\title{
Smoke Image Recognition Based on Local Binary pattern
}

\author{
Tiantian Tang ${ }^{1, a}$, Linhan Dai ${ }^{1, b}$, Zhijian Yin ${ }^{1, c, *}$ \\ ${ }^{1}$ College of Communication and Electronics, \\ Jiangxi Science \& Technology Normal University, \\ Nanchang, Jiangxi, China. \\ acotang@163.com; bdailinhan9619@163.com \\ cyinzhijian@jxstnu.edu.cn \\ *corresponding author
}

Keywords: background subtraction, local binary pattern (LBP), support vector machines (SVM)

\begin{abstract}
Smoke accurate detection, for the real-time fire detection and early warning has an important role. In order to overcome the problem that the smoke is low when the fire is burned, the video smoke detection method based on the local binary mode is proposed under the condition of disturbing the wind speed and other factors. In this method, the motion region is extracted by the background subtraction method, and each piece of motion area is processed to obtain local information. Then, the texture feature of each block is extracted by using the local binary model. Finally, the texture features of the smoke texture are used to obtain the texture feature. To achieve smoke image extraction. Finally, the support vector machine is used to classify the extracted features. Experiments show that the local binary pattern texture features show good attribute characteristics in the texture, and the correlation test data and the comparison result show that the texture feature is effective for the detection of smoke.
\end{abstract}

\section{Introduction}

With the popularity of large-scale building such as steel structure, the traditional gas-sensitive, temperature-sensitive, smoke-type, photosensitive and other fire detectors can not solve the contradiction between sensitivity and high reliability, easy to work environment dust, static , Electromagnetic interference, frequent fire leakage and false positives. In order to prevent and avoid the sudden and spreading of large space fire accidents as soon as possible, it is of great practical significance to explore timely and accurate fire detection methods. Image-based fire smoke detection technology is the use of cameras to monitor the scene, through the early fire smoke in the image to show the static and dynamic characteristics to detect the fire. In recent years, many scholars have studied the problem of image fire smoke recognition from different angles. Chen et al. [1] used the color feature statistics to find that the gray smoke color of the RGB3 channels was similar to finding the smoke fog set up for the condition. YH Yi et al [2] used the characteristics of smoke texture, the method of wavelet transform and BP neural network is used to identify and classify fire smoke images. These smoke recognition algorithms use artificial neural network and fuzzy logic technology to fuse multi-sensor signals, real-time poor. Based on the smoke vision, this paper proposes a smoke image detection method based on local binary model. Firstly, the background difference method is used to extract the motion area, and then the texture features of each block are extracted by LBP. Finally, the intrinsic relationship of the smoke image texture is used to obtain the intrinsic relationship of the smoke image texture, and the feature classification is carried out by using the support vector machine can correctly distinguish between smoke and non-smoke images. The experimental results show that the method has a high recognition rate. 


\section{Methods}

\subsection{Suspected Area Detection of Smoke Based on Background Subtraction Method}

The background subtraction method is a method of detecting moving objects using the current frame and background reference model comparison in the image sequence, and its performance depends on the background modeling technique used. In the motion target detection based on the background subtraction method, the modeling and simulation of the background image directly affect the detection effect. Regardless of any motion target detection algorithm, as far as possible to meet any image scene processing requirements, but due to the complexity of the scene, unpredictability, and the existence of a variety of environmental interference and noise, such as sudden changes in light, the actual background image In some objects of the fluctuations, the camera jitter, moving objects into and out of the scene on the impact of the original scene, making the background modeling and simulation becomes more difficult.

Background subtraction method is the principle of the current frame and the background image to get the difference between the moving target area, this method is better than the frame difference method to identify and extract the moving target, is the current motion segmentation One of the most commonly used methods. But need to build a background image, the background image must not contain moving objects, and should be able to constantly update to adapt to the current background changes, there are many ways to build background images, more commonly used based on a single Gaussian model of the background, Background construction based on mixed Gaussian model, background structure based on median filter, background structure based on Kalman filter, background model construction based on kernel function density estimation [3].

The algorithm first selects the average of one or several images in the background as the background image, and then subtracts the background image from the background image and the background image. If the number of pixels obtained is greater than a certain threshold, it is determined that there is a moving object in the monitored scene, thereby obtaining a moving target [4].

Background subtraction method is another idea of moving target recognition and segmentation in still background. The video frame image I $(x, y, t)$ can be regarded as the background image $b$ ( $x, y$, t) and the moving object $m(x, y, t)$ without considering the influence of the noise $n(x, y, t)$ composition, image $\mathrm{I}(\mathrm{x}, \mathrm{y}, \mathrm{t})$ is as follow:

$$
I(x, y, t)=b(x, y, t)+m(x, y, t)
$$

From the formula (1) we can get the moving target $\mathrm{m}(\mathrm{x}, \mathrm{y}, \mathrm{z})$ as follow:

$$
m(x, y, t)=I(x, y, t)-b(x, y, t)
$$

In practice, due to the influence of noise, the difference image $\mathrm{d}(\mathrm{x}, \mathrm{y}, \mathrm{t})$ composed of the moving target region and the noise can not be obtained by the equation (2) as follow:

$$
d(x, y, t)=I(x, y, t)-b(x, y, t)+n(x, y, t)
$$

Get the target of motion needs to be based on a certain principle of further processing, the most commonly used method is the threshold segmentation method, the formula is as follow:

$$
m(x, y, t)= \begin{cases}I(x, y, t) & d(x, y, t) \geq T \\ 0 & d(x, y, t)<T\end{cases}
$$

Where $\mathrm{T}$ is a threshold.

\subsection{Characteristics of smoke extraction and smoke judgment}

According to the characteristics of the static smoke image displayed on the image at the beginning of the fire, the texture feature of the smoke image is selected as the basis for the identification of fire smoke.

Texture is formed by the alternating gray scale in the spatial position, so there must be a relationship between the pixels of a pair of images in a certain spatial position, where the local 
binary model can better reflect this space relationship.

Local binary pattern (LBP) was first proposed by Ojala et al. [5]. The basic idea is to set the gray value of the central pixel as the threshold, and the pixel in its circular neighborhood is compared with the binary The LBP texture feature is invariant to the LBP texture feature when the gray scale of the image changes uniformly, so that the LBP texture feature is invariant. It is simple to calculate, has strong classification ability, and has significant effect in describing texture feature extraction. The LBP describes the texture of the image using the joint distribution $T=t\left(g_{c}, g_{0}, g_{1}, \ldots, g_{P-1}\right)$ of P pixels on each of the pixels and its circular neighborhood of R. (P, R), the LBP operator is not the same, and Figure 1 shows the gray value of the center of the local neighborhood, and $g_{p}(p=0,1,2, \ldots, P-1)$ corresponds to the gray value of the $\mathrm{P}$ equal points on the ring with radius $\mathrm{R}$. For 3 different LBP operators.

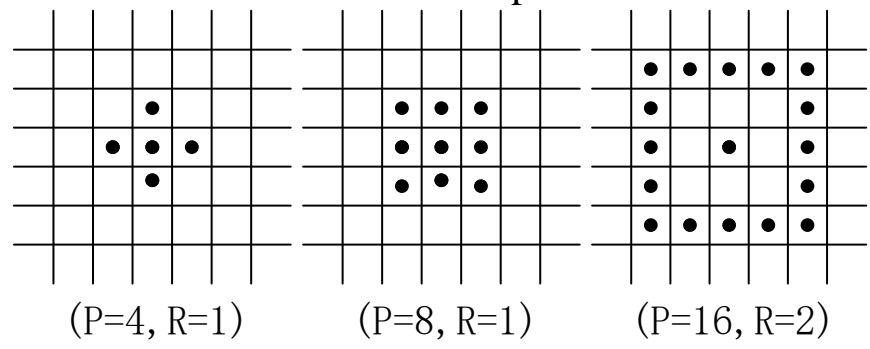

Figure 1.

In order to achieve the invariance of the gray scale of the texture operator, the gray value of the center point is subtracted from the gray value $g_{p}(p=0,1,2, \ldots, P-1)$ of the algebraic points on the annular neighborhood, and the joint distribution $\mathrm{T}$ is transformed into

$$
T=t\left(g_{c}, g_{0}-g_{c}, g_{1}-g_{c}, \ldots, g_{P-1}-g_{c}\right)
$$

Assumptions $g_{c}$ and $g_{p}$ independent of each other, equation (1) is roughly decomposed into, the formula as follow:

$$
T \approx t\left(g_{c}\right) t\left(g_{0}-g_{c}, g_{1}-g_{c}, \ldots, g_{P-1}-g_{c}\right)
$$

In the formula (6), $t\left(g_{c}\right)$ describes the gray distribution of the entire image, which has no effect on the local texture distribution of the image Therefore, the image texture feature can be described by the differential joint distribution, that is,

$$
T \approx t\left(g_{0}-g_{c}, g_{1}-g_{c}, \ldots, g_{P-1}-g_{c}\right)
$$

When the illumination of the image occurs additive, the relative size of the pixel gray value of the center pixel and its annular neighborhood is not changed, that is, $g_{p}-g_{c}$ is not affected by the change of the light additive Therefore, the difference between the center pixel and the neighborhood pixel The symbolic function of the value represents the texture of the image instead of the specific value

$$
T \approx \mathrm{t}\left(s\left(g_{0}-g_{c}\right), \mathrm{s}\left(g_{1}-g_{c}\right), \ldots, \mathrm{s}\left(g_{P-1}-g_{c}\right)\right.
$$

where $\mathrm{s}$ is the symbolic function, the formula as follow:

$$
s(x)=\left\{\begin{array}{l}
1, x \geq 0 \\
0, x<0
\end{array}\right.
$$

The result of the joint distribution $\mathrm{T}$ is sorted by the specific order of the pixels in the circular neighborhood to form a $0 / 1$ sequence. In this paper, the right pixel of the central pixel is used as the starting pixel in the counterclockwise direction, Each element $s\left(g_{p}-g_{c}\right)$ gives the binomial factor $2^{p}$, which can represent the local spatial texture of the pixel as a unique decimal number, which is called the $L B P_{P, R}$, which is also the texture operator called the local binary pattern ( Local Binary Pattern), the $L B P_{P, R}$ can be calculated by formula (10) as follow:

$$
L B P_{P, R}=\sum_{p=0}^{P-1} s\left(g_{p}-g_{c}\right) 2^{p}
$$


The specific LBP texture feature calculation process shown in Figure 4, the left side of the template in Figure 4 threshold, so that the neighborhood pixels and the central pixel for comparison, greater than 0 set to 1 , less than 0 set to 0 , (10100101), and finally the corresponding decimal number (165) is calculated. The LBP texture feature value of the pixel is 165 , and the LBP characteristic value is obtained for each pixel in the image, and the image is obtained LBP texture features, as shown in Figure 2. Because the LBP texture feature at the edge of the image is less affected by the neighborhood, the original pixel gray value is retained for the pixel at the edge of the image.

\begin{tabular}{|c|c|c|}
\hline 97 & 212 & 27 \\
\hline 128 & 131 & 135 \\
\hline 173 & 49 & 236 \\
\hline
\end{tabular}$\longrightarrow$\begin{tabular}{|l|l|l|l|}
\hline 0 & 1 & 0 & $\begin{array}{c}\text { Binary mode } \\
10100101\end{array}$ \\
\hline 0 & & 1 \\
\hdashline 1 & 0 & 1
\end{tabular}$\quad \begin{gathered}\begin{array}{c}\text { Decimal number } \\
165\end{array} \\
\end{gathered}$

Figure 2.

Figure 2 Calculate the local binary pattern texture feature $(P=8, R=2)$

In 2000, Pietikinen et al [5]. Described in article how to extend the LBP feature to make it invariant. Let's take a circular window with $\mathrm{P}=8$ and $\mathrm{R}=1.0$ as an example to explain the rotation invariance of the LBP operator. The gray value of the center point is $\mathrm{g}_{c}$, and the 8 pixel gray values in the 8 neighborhood are $g_{p}=\left\{\mathrm{g}_{0}, g_{1}, g_{2}, \ldots, g_{P-1}\right\}$, and the gray value $\mathrm{g}_{p}$ in the circular neighborhood when the image is rotated in the circumference of the circle with $\mathrm{g}_{c}$ as the center $\mathrm{R}$. For example, we have one such window as shown figure 3.

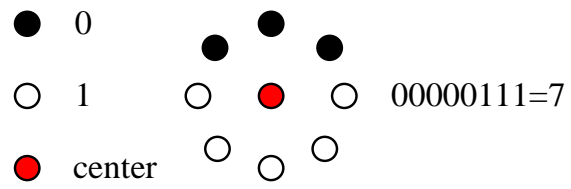

Figure 3.

The gray value $\mathrm{g}_{p}$ is moved on a circumference where the radius is $\mathrm{g}_{c}$ as the center.

We can find that as long as we take the above enumerated value of the minimum, you can eliminate the impact of rotation. For example, we get a binary LBP value of 10000011 (that is, the original anti-clockwise rotation of 45 degrees), and then continue to the right of the binary shift (plus its own, a total of eight possible value), then take the smallest. The result is the LBP value of the window.

When $\mathrm{p}=8, \mathrm{R}=1.0$, there will be $2^{p}=2^{8}=256$ values may be, while the rotation of the LBP feature will only exist 36 kinds of rotating binary mode:

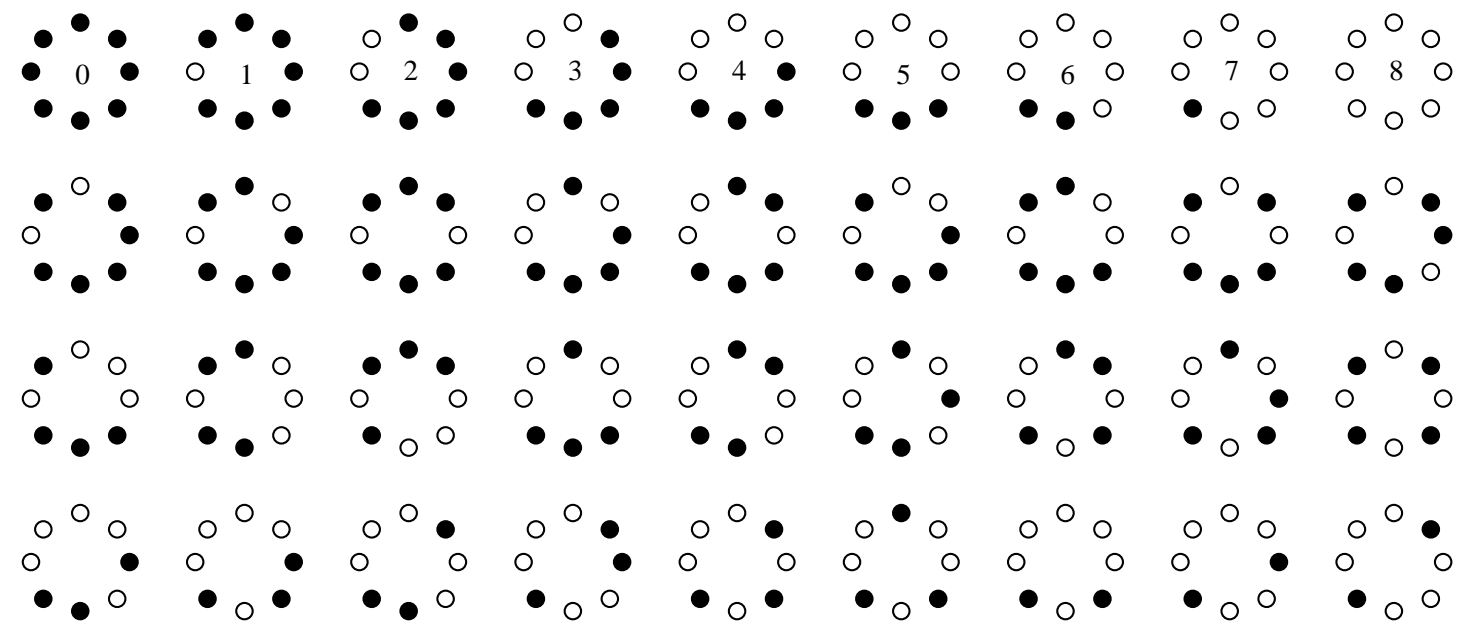

Figure 4

Later, T.Ojala found that the effect is not very good, because when we take the $\mathrm{P}$ and $\mathrm{R}$ values 
are large, it means that the local binary mode binary number will be very large ( $\mathrm{P}$ and $\mathrm{R}$ value of the larger, The more pixels in the neighborhood, T.Ojala has been given in the paper [5], P value of 24), so that the resulting histogram is very sparse, is not conducive to classification. And if $\mathrm{p}$ and $\mathrm{r}$ value is too small, it will make the angle of the cutting accuracy is reduced, so that the back of the classification performance. In order to solve these problems, T.Ojala et al.[5] proposed a method called "uniform pattern" to reduce the dimension of LBP features. T.Ojala et al. Found that there is a frequency in which a pattern appears in the image and its high, which is the uniform pattern, and they have a property that the number of black and white jumps is less than or equal to 2 (in the One line is the uniform model, the other is non- uniform mode). In addition, we can also introduce, in the $\mathrm{P}$ neighborhood, the number of equivalence mode $\mathrm{U}$ is: $\mathrm{U}=\mathrm{P} *(\mathrm{P}-1)+2$, other modes are called non-uniform mode. The LBP value of the uniform mode is equal to the number of 1 in the binary code, and the LBP of the non- uniform mode is $\mathrm{P}+1$, which is expressed by the formula:

$$
L B P_{P, R}^{\text {riu2 }}=\left\{\begin{array}{cl}
\sum_{p=0}^{P-1} s\left(g_{p}-g_{c}\right) & \text { if } U\left(L B P_{P, R}\right) \leq 2 \\
P+1 & \text { otherwise }
\end{array}\right.
$$

where $\mathrm{U}\left(L B P_{P, R}\right) \leq 2$ means that 0,1 times the number of times less than or equal to 2 . Although the equivalence model accounts for only a fraction of the total pattern, T.Ojala indicates that this fractional equivalence pattern can characterize more than $90 \%$ of the texture.

\section{Experimental results}

Using four common data of smoke and non-smoke images to do the experiment, all the image size is $48 * 48$ to do the feature extraction, Table 1 shows the four set 1 , set 2 , set 3 , set 4 data set Image situation. We are using the smallest number of data sets set 1 to do the training, the other three data sets set 2, set 3, set 4 to do the test.

The linear support vector machine (Lib-SVM) proposed by Chih-Jen et al. [6] is used as a classification. The SVM type is set to C-SVC. An effective additive nuclear approximation (AKA) [7] proposed by Vedaldi et al. Is used because the kernel enables fast training and testing of nonlinear cores and can be scaled to the data size. Since there is an imbalance between the number of smoke and non-smoking samples, we set the parameter weight to the ratio of the number of samples per sample to the number of samples.

\subsection{Evaluation standard}

To quantitatively compare our methods with the state-of-the-art algorithms, we define Detection Rate (DR), False Alarm Rate (FAR) and Error Rate (ERR) as follows:

$$
\begin{gathered}
\mathrm{DR}=\frac{P_{p}}{Q_{p}} \times 100 \% \\
\mathrm{FAR}=\frac{N_{p}}{Q_{n}} \times 100 \% \\
\mathrm{ERR}=\frac{Q_{p}-P_{p}+N_{p}}{Q_{p}+Q_{n}} \times 100 \%
\end{gathered}
$$

Where $\mathrm{Q}_{p}$ and $\mathrm{Q}_{n}$ represent the total number of positive and negative samples, $\mathrm{P}_{p}$ is the correct number of positive samples, and $\mathrm{N}_{p}$ is the number of negative samples that are classified as positive samples. Results The DR is actually classified as a positive sample rate (detection rate), and FAR is incorrectly classified as a positive sample rate (false positive rate). In other words, a good way is to get a high detection rate while getting a low false alarm rate and a low error rate.

In order to visually analyze the experimental results of the comparison of the methods on the three test sets, we show the results of each data set by plotting the DR and FAR tables, as shown in 
Table 1. It can be seen from Table 1 that the method of this paper has very good classification performance in smoke detection.

Table 1 Experimental result

\begin{tabular}{|c|c|c|c|c|c|c|c|c|c|c|}
\hline \multirow{2}{*}{\multicolumn{2}{|c|}{ Methods }} & \multicolumn{3}{|c|}{ set2 $(\%)$} & \multicolumn{3}{|c|}{$\operatorname{set} 3(\%)$} & \multicolumn{3}{|c|}{ set4 $(\%)$} \\
\hline & & DR & FAR & ERR & DR & FAR & ERR & DR & FAR & ERR \\
\hline \multirow{4}{*}{ LBP } & U2 & 97.238 & 3.182 & 2.99 & 96.411 & 2.409 & 2.651 & 96.229 & 2.176 & 2.515 \\
\hline & RI & 96.512 & 3.06 & 3.256 & 96.683 & 3.008 & 3. 071 & 95.652 & 2.38 & 2. 797 \\
\hline & RIU2 & 97.093 & 4. 284 & 3. 654 & 96.638 & 3. 372 & 3.37 & 96.096 & 2.942 & 3. 146 \\
\hline & 0 & 97.093 & 4. 039 & 3.522 & 96.638 & 3.642 & 3.585 & 96.273 & 2.942 & 3. 108 \\
\hline \multicolumn{2}{|c|}{ HOG } & 73.692 & 14. 198 & 19. 734 & 74.33 & 11.409 & 14. 339 & 74. 312 & 11.419 & 14.449 \\
\hline
\end{tabular}

\section{Conclusion}

This paper mainly studies the sensitivity of LBP to the texture characteristics of smoke in different mapping modes. Firstly, the background difference method is used to find the difference between the foreground and the background of the image, and the foreground graph is obtained. Then the texture feature histogram of the smoke is obtained by LBP texture feature. Finally, the result is obtained by SVM classifier. By comparing with other algorithms, the robustness of the algorithm is verified, which shows that the method is effective to extract the texture features of smoke images.

\section{Reference}

[1] Chen, T.H., et al. The Smoke Detection for Early Fire-Alarming System Base on Video Processing. in International Conference on Intelligent Information Hiding and Multimedia. 2006.

[2] Cui, Y., H. Dong, and E. Zhou. An Early Fire Detection Method Based on Smoke Texture Analysis and Discrimination. in Congress on Image and Signal Processing, Vol. 2008.

[3] Horprasert, T., D. Harwood, and L.S. Davis, A statistical approach for real-time robust background subtraction and shadow detection. IEEE Frame Rate Workshop, 1999: p. 1--19.

[4] Piccardi, M. Background subtraction techniques: a review. in IEEE International Conference on Systems, Man and Cybernetics. 2005.

[5] Ojala, T., M. Pietikäinen, and T. Mäenpää, Gray Scale and Rotation Invariant Texture Classification with Local Binary Patterns. IEEE Transactions on Pattern Analysis \& Machine Intelligence, 2000. 1842(7): p. 404-420.

[6] Chang, C.C. and C.J. Lin, LIBSVM: A library for support vector machines. 2011: ACM. 1-27.

[7] Qi, X., et al., Pairwise Rotation Invariant Co-Occurrence Local Binary Pattern. IEEE Transactions on Pattern Analysis \& Machine Intelligence, 2014. 36(11): p. 2199. 